\title{
Self-Efficacy and Its Relationship with Social Skills and the Quality of Decision-Making among the Students of Prince Sattam Bin Abdul-Aziz University
}

\author{
Salama Aqeel Al-mehsin ${ }^{1}$ \\ ${ }^{1}$ Prince Sattam Bin Abdul-Aziz University, Saudi Arabia \\ Correspondence: Salama Aqeel Al-mehsin, Prince Sattam Bin Abdul-Aziz University, Saudi Arabia
}

Received: February 27, 2017

Accepted: March 30, 2017 Online Published: June 27, 2017

doi:10.5539/ies.v10n7p108

URL: https://doi.org/10.5539/ies.v10n7p108

\begin{abstract}
The present study aimed to reveal the self-efficacy and social skills and their relationship to the quality of decision-making at Prince Sattam bin Abdulaziz University students, and determine the extent of the contribution of self-efficacy and social skills to the quality of decision-making. To achieve this, a questionnaire was built to identify self-efficacy, and a questionnaire of social skills, and a questionnaire of decision- making.The study sample was (560) female students from the College of Education in Prince Sattam bin Abdul Aziz University, the study results indicated that the self-efficacy of the study sample was moderate and that the relationship between self-efficiency and social skills and the quality of decision-making was a positive.

The findings revealed that the quality of decision-making interpreted about $81.5 \%$ of social skills, and it showed a positive statistically significant effect for the quality of decision-making on social skills, and the quality of decision-making interpreted about $69 \%$ of self-efficacy, and results also showed a statistically significant positive impact for the quality of decision-making on self-efficacy.
\end{abstract}

Keywords: self-efficacy, social skills, the quality of decision-making, Prince Sattam Bin Abdul-Aziz University

\section{Introduction}

The individual passes in his daily life positions require him to make a decision or more, The process of decision-making is an important process in every moment of our lives, which requires mastery of the individual to some of the skills that help in decision-making such as social skills, which is an essential requirement and a means help the individual to communicate and engage in social interactions with others, success in making many of the decisions depends on how the individual confidence in his ability and self-efficacy, which help him to go ahead on those decisions. self- efficiency contribute in determining the initiative behavior on him, it is also linked to social skills, which may affect the quality of decision-making, where the individuals who are characterized by high self-efficiency and high social skills tend to take decisions with high quality more than individuals with low self-efficiency and low social skills, in other words, when the ability is equal between some individuals, those who believe in their ability to do the work, they are more likely achieve it more successfully than those who do not believe so (Bandura, 1986).

Hence, people perceive self-competence affect the plans that they consider in advance and the decisions they make, and the beliefs of the individual about his self-efficacy effect on the choice of his behavior (Zayat, 2001). This is clear through his tendency to engage in tasks that feel a high degree of potential competition, while individuals who have belief of low self- efficiency with an actual rise in skill, perhaps suffering from a sense of weak self-confidence, and weakness in social skills, and the ability to make informed decisions.

Add to that the self-efficacy determines the amount of effort made by the individual and its average, and the extent of his commitment to continuing or perseverance in the performance of the target activity. The perseverance link with high level of self-efficacy, leads to a high performance, which dates back again to rise affects the level of self-efficacy, and thus affects the social skills and the quality of decisions that may be taken of the individual (Bandura, 1986). Self-efficacy may be considered a factor expresses the positiveness, which means that the students during the Undergraduate are in an urgent need for positive action and the active search for appropriate solutions to problems and try to invest available resources they have to satisfy their personal and social needs, and well-organized their needs and desires, according to their own priorities and its importance for 
them (Shell, Colvin, \& Bruning, 1995).

From here, the social skills move behavior and orientation towards positive interaction with others, and this comes from what the individual learn and acquire through social interaction, where the individual develops social skills or make it sufficient through models that he lives with in his life, or the ways and methods that he responds to the behavior of others, and here it was the duty of the education to provide some social skills necessary for the individual to live a quiet life (1983, Harter).

It could be argued that social skills represent the individual's ability to acquire different types of behaviors, and give an appropriate response, during the interaction with the environment, the tasks are represented in the performance of the required tasks easily, and social skills describes the individual's efficiency of social skills, and do a social activity requires from him skills to align what the other does and what he does, to correct his social activity path to achieve by that this harmonization, which may reflect positively or negatively on the ability to make decisions, as well as the affected of individual's self-efficiency in accordance with those decisions (Hoy \& Miskel, 2005 ). The attention to the development of decision-making skills among students would help the individual interactions with the community effectively, and is the responsibility of teaching individuals and how they reach to sound decisions on the responsibility of educational institutions, and the tutorial program in the universities considered of the most important means of achieving this principle, and it needs a study environment of friendliness so the student takes advantage of course at most. (Abu Hatab, 2007).

Moreover, the decision-making process requires the availability of the social environment in which the individual lives, the sum of skills available in the decision-maker, as well as the required objectives to achieve behind the decision making. (ElDesoki, 1998) It can be said that self-efficacy has a positive impact on social skills, which is to deal with others in the community, as well as decision-making contribute to effective role in providing psychological support to the individual. In spite of the difficulties faced by the students, some trying to search for causes that help in decision-making and the procedural definition of social skill is the total score you get on the scale used in this current study. The current study tried to identify the level of self-efficacy and social skills and their relationship to the quality of decision-making.

\subsection{Problem of the Study and Its Importance}

Undoubtedly, there are relationships among the scientific and intellectual developments and the many social changes, and the extent of an individual's ability, and his perceived self-efficiency and social skill in making its decisions. In this sense, the role of the educational authorities in the ability to develop educational plans and programs by working to raise the level of self-efficacy through knowing how they relate to a number of factors, such as social skills and its ability to influence the quality of decision-making among the individuals through the provision of training courses that will enable them refine their personalities, and thus the ability to exploit this development and increase self-efficiency and the development of social skills.

In spite of the challenges faced by university students, some of them are happy to search for the causes that lead them to make the decision, and here it is clear that the method of decision-making is important, and vary from learner to another and taken in itself may put pressure on individuals may amount to make him make false decisions. Based on the above, generate a sense of the need to identify the level of self-efficacy and social skills and their relationship to the quality of decision-making among a sample of students from the Faculty of Education at Sattam bin Abdulaziz University.

\subsection{Questions of the Study}

Specifically, the present study has tried to answer the following questions:

1) What is the level of self-efficacy among a sample of students from the Faculty of Education at Sattam bin Abdulaziz University?

2) What is the nature of the relationship between self-efficacy and all of the social skills and the quality of decision-making among the study sample?

\subsection{Significance of the Study}

The importance of the present study in an attempt to detect the nature of the relationship between the variables of the current study, as it seeks to contribute to the enrichment of an important aspect of the psychological and social studies field, which is the self-efficacy and social skills and knowledge of the nature of the relationship between them in taking the decision among the Education college students in Prince Sattam bin Abdul-Aziz University. The importance of the study came from the scarcity of studies and research that examined the level of self-efficacy and social skills and their relationship to the quality of decision-making. In addition, this study 
provides an objective measurement tools to measure self-efficacy and social skills and the quality of decision-making for college students can be used in research and related studies. And facilitate the proper educational guidance process, which must be taken into account when developing future plans and educational programs, both within the courses and plans of the school, or within an independent training programs; and then the understanding of the determinants of self-efficacy and social skills and the quality of decision-making contributes positively to resolve these problems and develop appropriate solutions for many of the behavioral, emotional and academic problems, which will benefit large to those who are in need of such programs in various scientific, personal and academic fields of study, behavioral and emotional.

\subsection{Procedural Definition of Terms}

The main concepts in the current study will be defined procedurally, namely:

1) Self-efficacy: means the Knowledge based on self-contain Resume predictions about a person's ability to overcome the various tasks in a successful way and these expectations are considered dimension of personal dimensions, and is represented in a self-convictions in the ability to control the requirements and overcome the difficult problems faced by the individual, and so through its own actions in the light of the environmental determinants menu. And self-efficacy is defined procedurally with the total score obtained by the screened on self-efficacy used by the researcher in this study.

2) Social skills: an individual's ability to interact positively with colleagues and good expression of positive and negative emotions, and control his emotions in the positions of social interaction with colleagues, as well as to identify the feelings of his colleagues and their hints, good disposition to suit the situation, measured by the score obtained by the student on the scale used in this study.

3) The quality of decision-making: the ability to make a good positive decision that achieves the set goals at the lowest cost and in a timely manner. It is defined procedurally by the score obtained by the student by answering the paragraphs of the quality of the decision-making scale.

\subsection{The Limits of the Study}

The results of the study are determined by the subject of self-efficacy and social skills and their relationship to decision-taking among the students of the Faculty of Education in Prince Sattam bin Abdul Aziz University in Al-Kharj City, as it is also determined by the following variables: specialization, gender. The results of the study are also determined in the light of the study tools developed by the researcher, namely: self-efficacy scale, social skills scale, and decision-making scale, and in light of what is available for each of the scales of validity and reliability, and thus disseminate the results of the study in the context of the current study limits, and similar communities.

\section{Literature Review}

Many researchers conducted studies about self-efficacy and the researcher reviewed some of these studies as follows

Haseeb (2001) conducted a study about the relationship between social skills and self-efficacy, between males and females and between elder and younger enacted in social skills and self-efficacy. He chose the study sample of (188) students from the university level, and the results indicated the existence of a correlation between some of the social skills and self-efficacy, and no differences between males and females in social skills, while suggesting the existence of differences in social skills between younger and older in favor of older. As Cruz (2002) also conducted a study aimed to explore the relationship between self-efficacy and academic achievement. The study sample consisted of (107) students from the University of Hawaii students). The results showed the existence of a positive correlation between self-efficacy and academic achievement, the academic achievement of students with high self-efficacy is high compared to the achievement of students with low self-efficacy.

The Alinviei (2010) conducted a study aimed to determine the relationship between social skills and self-efficacy among outstanding and ordinary students at secondary schools in Jeddah, Saudi Arabia. Where the study sample consisted of 200 students from secondary school students, and the results indicated the presence of positive correlation between the degree of social skills with its different dimensions and between the scores of self-efficacy among talent and ordinary secondary school students alike. To find out the expectations of self-efficacy in both self- confidence and social skills for university students, Eid (2012) held a study its sample consisted of (124) students from the University of Qassim, Saudi Arabia, the researcher used the self-efficacy scale, self-confidence scale, social skills scale, the study results indicated the presence of a positive correlation between expectations of self-efficacy and social skills. 
Yousuf (2013) held a study that aimed to determine the relationship between social skills and self-efficacy and academic achievement among middle school students in Hail, Saudi Arabia. The study sample consisted of (290) female and female students, the study results indicated the presence of correlation coefficient of the relationship between social skills and self-efficacy and achievement among members of the study, the results also indicated the presence of significant differences in social skills attributed to gender and in favor of females, and to the existence of significant differences in the level of self-efficacy attributed to gender in favor of males. To measure the degree of possessing the university students of social skills and whether that degree varies depending on the variables of gender and specialization Said (2014) conducted a study where its sample consisted of (485) female and female students, the researcher used the social skills scale. The study results indicated the presence of a statistically significant differences on the total score of students' possession of the social skills due to the gender variable, and so in favor of females, and no differences due to the variable of specialization, and the results showed also that the variables of gender and specialization interpret together about $24 \%$ of the variation degrees of students' possession of social skills.

Based on the above, it can be said that the results of studies on self-efficacy and social skills have shown that there is a relationship between self-efficacy and social skills by having a correlation evidenced by the studies of both Eid (2012) and Yousuf (2013), Haseeb (2001) and Alinviei (2010) and no studies were conducted on the variables with the decision, which the current study seeks to verify. The results of previous studies such as Otaibi (2010) also showed the existence of an effect of a number of variables, most notably (the positive correlation coefficient between degrees of ability for decision-making and the effectiveness of the self), while the results of Cruz (2002) showed a positive correlation between self-efficacy and academic achievement.

\section{Methods and Procedures}

\subsection{Study Approach}

Descriptive method was used in order to achieve the objectives of the study.

\subsection{The Study Population and Its Sample}

The study population consisted of all students in the Faculty of Education in Prince Sattam bin Abdul Aziz University in the levels of the undergraduate students at first, second, third and fourth years and in the majors (Islamic Studies, kindergarten and special education) regular in attendance official hours for the academic year 2015/2016 and their number (2800) students. The study sample consisted of (560) students who were studying in the first, second, third and fourth year levels in (bachelor's) degree in Prince Sattam bin Abdul Aziz University, where they were chosen randomly cluster. This sample formed $(5 \%)$ of the total number of members of the community.

\subsection{Study Tools}

The researcher used the following tools:

\section{First: Self-efficacy Scale}

The scale was built after reviewing the tools used in previous studies and theoretical frameworks in this area, and the scale consisted of ten paragraphs listed on the ladder of five alternatives are (rarely, sometimes, often, always, never). The responder responds on each paragraph by choosing one of these alternatives by putting a reference in front of the paragraph and under the alternative that he deems appropriate for his convictions. The alternatives are given the following grades $(5,4,3,2,1)$ respectively.

\subsubsection{Content Validity}

The scale was displayed on a number of arbitrators from the faculty members in the Department of Educational Psychology in the Faculty of Education, Prince Sattam bin Abdulaziz University. They were asked to judge how the representation of the paragraphs of the self- efficiency and the safety of the language of paragraphs, and any other comments they deem appropriate, and the ration $(80 \%)$ and above was adopted to keep the paragraph and considered appropriate, and it turned out from the arbitrators estimates that all the paragraphs measure self-efficacy, the language was modified to some paragraphs.

\subsubsection{Reliability of The Scale}

The indications of reliability of the scale was measured by using the test/re-test method for reliability of the scale; where the test was applied twice and interval time amounted two weeks on a sample of (60) students from outside the study sample, and the value of the correlation coefficient between the scores of students in the two applications (0.83). As Cronbach's alpha coefficient was extracted as an indicator of the internal consistency of the scale, using the previous experimental sample, the results showed reliability coefficient of $(0.81)$. 


\subsubsection{Self-Efficacy Scale Correction}

In assessing the degree of this scale grading, the existing alternatives were used, namely, (rarely, sometimes, often, always, never) so that grades are given to alternatives $(5,4,3,2,1)$, respectively, and therefore the maximum degree can be obtained by the respondent on the scale is (50) degrees, while the inferior degree can be obtained by the respondent is (10) degrees.

Second: social skills scale

After the researcher reviewed some social skills scales that some of the studies included (Miller, 1995; Merrell, 1993; Greesham \& Elliott, 1990, Attar, 2007); he developed a scale of social skills; to suit the sample of the current study, since the scales that seen by the researcher carried out on non-Saudi environments, some other ones were developed to suit non-university students samples, and the scale in its final form consisted of (22)items distributed among three dimensions, will be described: this standard determines the behaviors and there are no negative items.

\subsubsection{Content Validity}

The scale was displayed to a number of arbitrators from the faculty members in the Department of Educational Psychology in the Faculty of Education, Prince Sattam bin Abdulaziz University. They were asked to judge how the representation of the paragraphs of social skills and the integrity of the language of the items, and the extent of affiliation of the item to the dimension and any other observations they deem appropriate, the $(85 \%)$ and above was adopted to keep the paragraph and considered appropriate, and it turned out from the arbitrators estimates that all paragraphs measure social skills, the language of some paragraphs was modified. And so the scale of the social skills after a presentation to the arbitrator consisted of (22) paragraph covers three dimensions: the cooperation dimension: ( 7 items) and includes behaviors such as seeking help from others, participation and committed to instructions, and the work habits dimension: (9 items) and includes initiative behaviors like ask others for information, and to present himself to others and respond to the actions of others appropriately, and the self-tuning dimension: (6 items) and includes behaviors such as respond appropriately when harassed by others, and to act in the situations where he involves and need to reconcile or to take different views.

\subsection{Correction Method}

The answers of the study members were corrected as follows: Each paragraph of the scale ladder answers consists of five scales: always given (5) degrees, usually given (4) degrees, sometimes given (3) degrees, rarely given (2) degrees, and never given (1) degree. And thus a higher degree can be obtained by the responder is (110) by $(5 \times 22)$ and the lowest score is $(22)$ degrees by $(1 \times 22)$.

\subsubsection{Third: The Quality of Decision-Making Scale}

After consulting a group of Arab and foreign educational studies related to this subject, and take advantage of them, the researcher prepared a scale of quality of decision-making. Consisting of (20) items half of it positive, and the other half is negative. He used gradient Quintet (applies largely applies moderately and apply a low degree would never apply) representing items response to each paragraph, and this scale is applied to age groups ranging from (19-24).

\subsubsection{Content Validity}

The scale has been displayed on a number of arbitrators from the faculty members in the Department of Educational Psychology in the Faculty of Education, Prince Sattam bin Abdulaziz University. they were asked to judge how the representation of the items of the quality of decision-making and the integrity of the language of paragraphs, and any other comments they deem appropriate, and the ratio (80\%) and above was adopted to keep the paragraph and considered it appropriate, and it was clear from the arbitrators' estimates that all the items measure self-efficacy, the language of some paragraphs was amended.

\subsubsection{Reliability of the Scale}

To ensure the reliability of the scale, the researcher apply the scale on a pilot sample of (60) students from the Faculty of Education at Sattam bin Abdulaziz University, the reliability coefficient of the scale reached (0.89) and the second by re-application of the scale on the previous sample in a retail midterm way, where the correlation coefficient of the scale as a whole was calculated, the calculated reliability coefficient was $(0.91)$. This value was considered appropriate for the purposes of the study.

Correction Method:

The answers of the members of the study were corrected as follows: Each item of the scale ladder answers 
consists of five scales: always given (5) degrees, usually given (4) degrees, and sometimes given (3) degrees, and rarely given (2) degrees, and never given (1) degree. And thus a higher degree can be obtained by the responder $(100)$ by $(5 \times 20)$ and the lowest score is $(20)$ degrees by $(1 \times 20)$. The arithmetic means of the scale of quality of decision-making ranking as follows: high (more than 3.66), medium (more than 2.33 - 3.66); low (less than 2.33)

\subsection{Statistical Treatment}

To answer the first question, which is: What is the level of self-efficacy among a sample of students in the College of Education at Sattam bin Abdulaziz University? Frequencies, percentages, averages, and standard deviations of the responses of the study sample as a whole on self-efficiency scale items were extracted.

To answer the second question, which is: What is the nature of the relationship between self-efficacy and all of the social skills and the quality of decision-making? ". Correlation coefficient between self-efficacy and existence of decision-making and social skills and the quality of decision-making were extracted, as the use of multiple regression analysis to determine the nature of the relationship between these variables.

\section{Results of the Study}

Results of the first question, which states: What is the level of self-efficacy among a sample of students in the Faculty of Education at Sattam bin Abdulaziz University:

To answer this question arithmetic means and standard deviations of the level of self-efficacy among a sample of students in the Faculty of Education at Sattam bin Abdulaziz University, and the table below illustrates this:

Table 1. The arithmetic means and standard deviations of the items of the level of self-efficacy among a sample of students in the Faculty of Education at Sattam bin Abdulaziz University in descending order according to means

\begin{tabular}{|c|c|c|c|c|c|}
\hline Rank & $\mathrm{N}$ & Items & Mean & $\begin{array}{l}\text { Standard } \\
\text { deviation }\end{array}$ & Level \\
\hline 1 & 7 & $\begin{array}{l}\text { I do not care about what happens to me from problems at all, because I can } \\
\text { get rid of them simply. }\end{array}$ & 4.15 & .822 & High \\
\hline 2 & 8 & I find a solution to every problem I face effectively. & 4.13 & .901 & High \\
\hline 3 & 9 & When I face a new problem, I know how to deal with it. & 4.11 & .799 & High \\
\hline 4 & 10 & $\begin{array}{l}\text { When someone puts me in front of a problem, I know how to get rid of it } \\
\text { easily, because I own several ideas that help me to solve it. }\end{array}$ & 4.10 & .896 & High \\
\hline 5 & 3 & It is easy for me to achieve my goals and my intentions. & 3.65 & 1.031 & Moderate \\
\hline 6 & 2 & I can solve difficult problems if strained myself enough & 3.64 & 1.189 & Moderate \\
\hline 7 & 1 & If someone Opposed me, I can find ways and means to achieve what I & 3.63 & .992 & Moderate \\
\hline 8 & 6 & I look at the difficulties simply, so I continuously relay on my own & 2.53 & 1.117 & Moderate \\
\hline 9 & 4 & If am surprised by unexpected situations, I know how to act. & 2.52 & 1.060 & Moderate \\
\hline \multirow[t]{2}{*}{10} & 5 & $\begin{array}{l}\text { I think that I am unable to handle difficult problems appropriately even if } \\
\text { it was a surprise. }\end{array}$ & 2.52 & 1.356 & Moderate \\
\hline & & Self-efficacy scale & 3.50 & .506 & Moderate \\
\hline
\end{tabular}

Table 1 shows that the means ranged between (2.52-4.15) where item No. (7) Which states, "I do not care what happens to me from problems at all, because I can get rid of them simply" came in the first place with a mean of (4.15), while items No. (4 and 5) which state "if I,m surprised by unexpected positions, I know how to act", and "I think that I am unable to handle difficult problems appropriately even if it was a surprise," came in the last rank and an arithmetic mean was (2.52).,he arithmetic mean for self-efficacy scale as a whole reached (3.50).

Second question: What is the nature of the relationship between self-efficacy and all of the social skills and the quality of decision-making?

To answer this question Pearson correlation between self-efficacy and all of the social skills and the quality of decision-making, was extracted and Table 2 illustrates this. 
Table 2. Pearson correlation coefficient of the relationship between self-efficacy and all of the social skills and the quality of decision-making

\begin{tabular}{|c|c|c|}
\hline Dimensions & & Self-efficacy scale \\
\hline \multirow{3}{*}{ Cooperation } & The correlation coefficient $\mathrm{R}$ & $.663^{* *}$ \\
\hline & Statistical significance & .000 \\
\hline & Number & 560 \\
\hline \multirow{3}{*}{ Work habits } & The correlation coefficient $\mathrm{R}$ & $.827 * *$ \\
\hline & Statistical significance & .000 \\
\hline & Number & 560 \\
\hline \multirow{3}{*}{ Self-tuning } & The correlation coefficient $\mathrm{R}$ & $.802 * *$ \\
\hline & Statistical significance & .000 \\
\hline & Number & 560 \\
\hline \multirow{3}{*}{ Social skills scale } & The correlation coefficient $\mathrm{R}$ & $.844 * *$ \\
\hline & Statistical significance & .000 \\
\hline & Number & 560 \\
\hline \multirow{3}{*}{$\begin{array}{l}\text { The quality of } \\
\text { decision-making scale }\end{array}$} & The correlation coefficient $\mathrm{R}$ & $.831 * *$ \\
\hline & Statistical significance & .000 \\
\hline & Number & 560 \\
\hline
\end{tabular}

* Statistically significant at the significance level (0.05).

** Statistically significant at the significance level $(0.01)$.

Seen from the Table 2 the existence of a positive relationship (proportional) statistically significant between self-efficacy and all of the social skills and the quality of decision-making, and regression analysis has been used and Table 4 and 5 shows that.

Table 3. The results of regression analysis to measure the impact of the quality of decision-making on social skills

\begin{tabular}{|c|c|c|c|c|c|}
\hline Independent variable & $\begin{array}{c}\text { Correlation coefficient } \\
\mathrm{R} \\
\end{array}$ & $\begin{array}{l}\text { Determination coefficient } \\
\text { R2 }\end{array}$ & B Coefficient & F value & Sig \\
\hline The quality of decision-making & .903 & .815 & .945 & 2453.982 & .000 \\
\hline
\end{tabular}

Dependent variable: social skills

It is clear seen from the above table that the interpreter disparity was $(0.815)$ which means that the quality of decision-making interpreted about $81.5 \%$ of social skills, it shows a statistically significant positive impact of the quality of decision-making on social skills, as the value of $\mathrm{F}$ was (2453.982) with a statistical significant (0.000), and the forecasting coefficient $\mathrm{R}$ was $90.3 \%$.

Table 4. Results of regression analysis to measure the impact of the quality of decision-making on self-efficacy

\begin{tabular}{|c|c|c|c|c|c|}
\hline Independent variable & $\begin{array}{c}\text { Correlation } \\
\text { coefficient } \\
\mathrm{R} \\
\end{array}$ & $\begin{array}{l}\text { Determination coefficient } \\
\text { R2 }\end{array}$ & B Coefficient & F value & Sig \\
\hline The quality of decision-making & .831 & 690 & .780 & 1244.550 & .000. \\
\hline
\end{tabular}

Dependent variable: self-efficacy

It can be seen from the above table that the interpreter disparity was $(0.69)$ which means that the quality of decision-making interpreted about $69 \%$ of self-efficacy, it shows a statisticaly significant positive impact of the quality of decision-making on self-efficacy, as the value of $\mathrm{F}$ was (1244.550) with a statistically significance (0.000), and the forecasting coefficient $\mathrm{R}$ reached (83.1\%).

\section{Discussion}

1) Discussion of the first question 
This result can be interpreted in the occurrence of most of the study sample in the standard medium of self-efficacy, that the self-awareness process is still in the process of growth and gain experience, as individuals at this stage did not reach the total self-reliance stage, they are at the stage of the construction of many skills that help them make the decision, they need to gain experience, which supports both direct and indirect information, and works to form positive trends for individual abilities and potential and consequently working to raise the individual's ability to cope with events and take positions on them.

Bandura (1986) indicated that self-perceptions and self-regulatory mechanisms are important in understanding the relationship taking place between environmental stimuli and successful behavior towardsit. As the self-organization and raise the level of self-efficacy process needs to expertise, knowledge and activities which help to self-awareness and the formation of self-system in all its aspects, and this is what it should be pointed out in this regard. And this result can be attributed to the classification of most of the study sample in the standard medium of self-efficacy and it is indicated by an individual for positions that require the ability to do behavior, and this depends itself on the level of self-efficacy of the individual and their responsibilities towards these behaviors and attitudes that requires him to rely the abilities and potential of self, and thus the ability to harmonious performance in various areas of life.

The result of this study is consistent with Muhsen (2006) study which revealed that the level of self-efficacy at Yarmouk University students, where most of the members of the sample was classified in medium level of self-efficacy. There were no results of other studies in this area to disagree with the current study. This requires the development of individuals in front of the experiences and attitudes of behavior, working to raise the level of their own competence, and by giving them the knowledge which they will act depending on what has been gained. The result of this study is unique in detecting the level of self-efficacy among students of the Faculty of Education.

2) Discussion of the second question, which states that: What is the nature of the relationship between self-efficacy and all of the social skills and the quality of decision-making?

The results related to the second question revealed that the quality of decision-making interpreted about $81.5 \%$ of social skills, and show a positive statistically significant effect for the quality of decision-making on social skills, and the researcher attributes the current result is that the social skills of the important elements that determine the nature of the everyday interactions of the individual with those around him in different contexts, which is efficient in the case of completeness of the manifestations of psychological and social compatibility (Shawki, 2004), and that social skills play an important role in the success of the individual in the establishment of an efficient social interaction with others and the ability to continue this interaction, the slide of these skills explains the failure experienced by some individuals in the positions of the process of life despite the rise of their mental capabilities, but it does not stand often at the limits of poor social interaction and lower social competence, and lack of effectiveness in the social environment for individuals who suffer from low social skills.

This is in the framework decision will be the start of the problem or to determine the target and gather information and search for alternative solutions and choose the best alternative and the end of the implementation, monitoring and evaluation. Not always an easy process. This is because the problems faced by decision makers during this many and complex, including stages of what is associated with self-configuration of the decision-maker and some of which is linked to the environment and the ocean with all its opportunities and public relations, traditions and other targets. And that the curricula and methods of decision makers are affected by personal factors stemming from their personalities and the most important of these factors. Attitudes, values and their level of scientific expertise and past pattern of behavior and social backgrounds and that these traits vary from person to person.

Add to that the results indicated that the quality of decision-making interpreted about $69 \%$ of self-efficacy, and show a statistically significant positive impact to the quality of decision-making on self-efficacy. And the current result comply with the results of Spencer's (1997) study, which indicated that there is a relationship between self-efficacy and decision-making, and the researcher believes that some of the university teaching staff members focus their attention to the aspects and ignore other aspects.

Since the subject of the decision- making is very important and needs from a teacher a high level of objectivity as the ability to make the right decisions as well as the urgent need for real knowledge constraints intervention exhibitor on the teaching process so not to give objective aspect its right, the decisions taken far from maturity will be effective solutions not achieved. Where the decision-making of the most dangerous tasks facing the teacher a process which must be conscious of identifying the problem and the challenges posed by the position variables and environment of decision- making represented in the number and the quality of people affected by 
the decision and the amount and quality of available information and how it is processed, and in the case of lack of knowledge of the real problem, the decision which will be taken will be improper totally because of its inadequate to the problem, which was released in question.

In spite of the challenges faced by university students, some of them are happy to search for the reasons that lead them to make the decision, while others are struggling with richer sources of information and diversity and the presence of potential latent they have, but they consult their peers or teachers or parents in order to make decisions in a way dims self-values and previous experiences.

\section{Recommendation}

In light of the findings of the current study, the researcher recommends the following: further studies aim to identify other variables associated with the quality of decision-making. The necessity to work to create an academic climate is reassuring because of its positive effect on the acquisition of the students various skills to establish development courses for students for the development of their level in the decision-making

\section{References}

Abu Hatab, G. (2007). The dynamics of the publication and promotion of social skill. Jordan, Amman, Dar Wael for publication.

Alinviei, M. (2010). Social skills and their relationship with self-effectiveness among talented and ordinary students from high school students in Jeddah (Unpublished MA thesis). Umm Al Qura University.

Al-Otaibi, B. (2010). Decision-making and its relationship to both self-efficacy and social support in a sample of students' mentors at Taif (Unpublished MA thesis). University of Umm Al-Qura.

Attar, I. (2007). Social skills and shyness and their relationship with students' achievement from different stages of learning Balmemklh Saudi Arabia. Journal of College of Education, Education and Psychology, 31(2), 57-84.

Bandura, A. (1986). Social Foundation of Thought and Action Engle Wood Cliffs Prentice Hall. Dissertation Abstract International, 56(4), 123-129.

Cruz, L. (2002). The Influence of Family Support Acculturation Ethnic Identity and Self- Efficacy on The Acadmic Achievement of Native Hawiian and Hawiian-Reared College Students. Dissertation Abstract International, 40(1), 255.

Eid, M. (2012). Expectations of self-efficacy and its relationship with self-confidence and social skills among university students in Saudi Arabia. Journal of the Faculty of Education, Mansoura University, 81.

El Desoki, H. (1998). Human behavior "between theory and practice". Cairo: Dar Arab culture.

Gresham, F., \& Elliott, S. (1990). Social Skills Rating System. Circle Pines, MN: American Guidance Service.

Harter, S. (1983). Developmental Perspective on the Self System. In P. Mussen (Ed.), Hand book of child psychology isocalization, personality and Social development. NY: John Wiley \& Sons.

Hasib, A. (2001). Social skills and effective self for Talented and ordinary university students. Journal of Psychology, 59. Retrieved from http://www.arabpsynet.com/Journals

Hoy, W., \& Miskel, C. (2005). Education Administration: Theory, research, and practice. New York: McGraw-Hill.

Merrell, K. W. (1993). Using behavioral rating scales to assess social skills and antisocial behavior in school settings: Development of the school social behavior scales. School Psychology Review, 22(1), 115-133

Miller, R. (1995). On the nature of embarrassability: Shyness, social evaluation and social skills. Journal of Personality, 63(2), 315-339. https://doi.org/10.1111/j.1467-6494.1995.tb00812.x

Said, B. (2014). Degree possession of good social skills among students of Teeba University in light of the variables of gender and specialization. Arab Studies in Education and Psychology, 45(2).

Shawqi, T. (2004). Social communication skills. Cairo, Dar Gharib for Publishing and Distribution.

Shell, D., Colvion, C., \&Bruning, R. (1995). Self-Efficacy, Attribution, and Out-come expectancy mechanisms in reading and writing achievement: grade-level and achievement-level difference. Journal of Educational psychology, 87(3), 386-398. https://doi.org/10.1037/0022-0663.87.3.386

Yousuf, R. (2013). Social skills and their relationship with the public and efficient self-achievement among a sample of students in middle school with a barrier in the light of a number of variables. Journal of the 
Islamic University for Educational and Psychological Studies, 21.

Zayat, F. (2001). Cognitive Psychology, Part II, entrances, models and theories (1st ed.). The publishing house of Universities, Egypt.

\section{Copyrights}

Copyright for this article is retained by the author(s), with first publication rights granted to the journal.

This is an open-access article distributed under the terms and conditions of the Creative Commons Attribution license (http://creativecommons.org/licenses/by/4.0/). 